\title{
INTERPRETATIONS INTO $p$-ALGEBRAS
}

\author{
O. CARTAGENA, C. GONZÁLEZ, R. LEWIN, AND O. RUBILAR
}

(Communicated by Louis J. Ratliff, Jr.)

\begin{abstract}
We prove that there is only one interpretation from the variety of $p$-algebras into itself.
\end{abstract}

\section{INTRODUCTION AND PRELIMINARIES}

The concept of interpretability of one variety into another was introduced by Neumann [4] in the study of Malt'sev conditions; it was later developed by García and Taylor [3]. Roughly speaking, a variety $V$ is interpretable in a variety $W$ if the operations of $V$ can be defined in terms of the operations of $W$ in such a way that the (underlying sets of the) algebras in $W$ with these new operations are algebras in $V$. For precise definitions the reader is referred to the above mentioned [3].

In what follows $\mathbf{D}_{\mathbf{0 1}}$ stands for the variety of distributive lattices with greatest and least elements. $\mathbf{B}_{\omega}$ stands for the variety of distributive pseudocomplemented algebras, or $p$-algebras. For background on these, check for instance [1].

A $p$-algebra is a universal algebra $\left\langle A ;+, \cdot,^{*}, 0,1\right\rangle$ such that

(1) $\langle A ;+, \cdot, 0,1\rangle \in \mathbf{D}_{01}$,

(2) $x(x y)^{*}=x y^{*}$,

(3) $0^{* *}=0$,

(4) $0^{*}=1$.

Remark. For any $a \in A, a^{*}$ is called the pseudocomplement of $a$. We can prove that $a^{*}$ is the greatest element $x$ such that $a \cdot x=0$.

Some identities verified in $\mathbf{B}_{\omega}$ that will be used in the sequel are the following:

Theorem. In $\mathbf{B}_{\omega}$ the following identities hold:

(i) $x \cdot x^{*}=0$,

(ii) $x \leq x^{* *}, x^{*}=x^{* * *}$,

Received by the editors January 23, 1990 and, in revised form, April 26, 1990.

1980 Mathematics Subject Classification (1985 Revision). Primary 06D15; Secondary 08B05.

The results reported here were obtained in a seminar conducted at Pontificia Universidad Católica de Chile-Sede Temuco during 1989. This was funded by FONDECYT project 241/88 and PUC-Temuco, project $1 / 88$. 
(iii) $(x+y)^{*}=x^{*} y^{*}$,

(iv) $(x y)^{*} \geq x^{*}+y^{*}$,

(v) $x^{* *} y^{* *}=(x y)^{* *}$.

In what follows we need to determine the free $p$-algebras in one and two generators, $\mathbf{F}_{\mathbf{B}_{\omega}}(\mathbf{x})$ and $\mathbf{F}_{\mathbf{B}_{\omega}}(\mathbf{x}, \mathbf{y})$. Using a result by Berman and Dwinger [2, p. 240], after some tedious computations, we can prove that $\mathbf{F}_{\mathbf{B}_{\omega}}(\mathbf{x})$ is the algebra whose elements are $0, x, x^{*}, x^{* *}, x+x^{*}, x^{*}+x^{* *}$ and 1 , and that $\mathbf{F}_{\mathbf{B}_{\omega}}(\mathbf{x}, \mathbf{y})$ is the algebra whose elements are the finite sums of the following set $S$ of terms

$$
\begin{aligned}
& S=\left\{0,1, x, x^{*}, x^{* *}, y, y^{*}, y^{* *}, x y, x y^{*}, x y^{* *},\right. \\
& x^{*} y, x^{*} y^{*}, x^{*} y^{* *}, x^{* *} y, x^{* *} y^{*}, x^{* *} y^{* *},(x y)^{*}, \\
&\left.\left(x y^{*}\right)^{*},\left(x^{*} y\right)^{*},\left(x^{*} y^{*}\right)^{*},(x y)^{*}\left(x^{*} y^{*}\right)^{*},\left(x y^{*}\right)^{*}\left(x^{*} y\right)^{*}\right\} .
\end{aligned}
$$

\section{INTER PRETING $\mathbf{D}_{01}$ into $\mathbf{B}_{\omega}$}

As stated in the introduction, we must define the operations $\vee, \wedge, \mathbf{0}$ and $\mathbf{1}$ of $\mathbf{D}_{\mathbf{0 1}}$ in terms of the operations of $\mathbf{B}_{\omega}$, that is, we need binary terms $\sigma(x, y)$ and $\pi(x, y)$, and constant terms $c_{0}$ and $c_{1}$ in the language of $p$-algebras such that given any $p$-algebra $A$, if we define

$$
\begin{aligned}
x \vee y & :=\sigma(x, y), \\
x \wedge y & :=\pi(x, y), \\
\mathbf{0} & :=c_{0}, \\
\mathbf{1} & :=c_{1},
\end{aligned}
$$

$\langle A ; \vee, \wedge, \mathbf{0}, \mathbf{1}\rangle \in \mathbf{D}_{\mathbf{0 1}}$.

It is immediate that the "trivial" and the "dual" interpretations will work, that is, define

$$
\begin{aligned}
& x \vee y:=x+y, \quad x \vee y:=x y,
\end{aligned}
$$

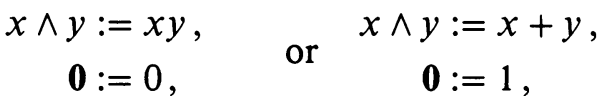

$$
\begin{aligned}
& \mathbf{1}:=1, \quad \mathbf{1}:=0 \text {. }
\end{aligned}
$$

In what follows we will prove that no other definition is possible.

Let us assume then that

$$
x \vee y=\sum t_{i}(x, y)
$$

for a finite set of terms $t_{i} \in S$.

Since $x \vee y$ is an idempotent operation, we must have that

$$
x=x \vee x=\sum t_{i}(x, x) .
$$


This implies that the terms $1,\left(x^{*} y\right)^{*},\left(x y^{*}\right)^{*}$, and $\left(x^{*} y\right)^{*}\left(x y^{*}\right)^{*}$ cannot appear in the right-hand side of (1) or else

$$
x=\sum t_{i}(x, x)=1 .
$$

In the same way the terms $x^{*}, y^{*}, x^{*} y^{*}$, and $(x y)^{*}$ cannot be summands of $\sigma$ or else

$$
0=0 \vee 0=1
$$

Similarly, if the terms $x^{* *} y^{* *},\left(x^{*} y^{*}\right)^{*}$, or $y^{* *}$ appear as summands

$$
x=x \vee x=\sum t_{i}(x, x) \geq x^{* *}
$$

which is not possible.

Notice now that the constants 0 and 1 must be defined in terms of the constants 0 and 1 so either $\mathbf{0}=0$ and $\mathbf{1}=1$ or $\mathbf{0}=1$ and $\mathbf{1}=0$.

In any case

$$
x \vee 0=0 \vee x \in\{x, 0\}
$$

and

$$
x \vee 1=1 \vee x \in\{x, 1\} .
$$

Evaluating $x^{* *} y^{*}, x^{*} y^{* *},(x y)^{*}\left(x^{*} y^{*}\right)^{*}$ in $(x, 0)$ or $(0, x)$ yields $x^{* *}$ so if these terms appear in $\sigma$, we would have $x \vee 0=0 \vee x \geq x^{* *}$ which is not possible by the remark above.

Similarly, evaluating $x^{* *} y, x y^{* *}, x^{*} y, x y^{*}$ in $(x, 1)$ or $(1, x)$ yields $x^{* *}$ or $x^{*}$, so if these terms appear in $\sigma$

$$
1 \vee x=x \vee 1 \geq x^{*} \text { or } x^{* *} \text {, }
$$

so

$$
x \vee 1=1 \vee x=1 .
$$

It is not difficult to check that, if this is the case, then both $x$ and $y$ must appear in $\sigma$ so the terms here considered are absorbed.

We are left with the terms $x, y$ and $x y$. Obviously if $x$ appears in $\sigma$, so does $y$ and if they both appear in $\sigma$, the third is absorbed. One of them must be present or else

$$
x \vee y=0
$$

which is not possible, so we have only two possibilities, either

$$
x \vee y=x+y \text { or } x \vee y=x y .
$$

It is easy to check that in these cases

$$
x \wedge y=x y, \quad \mathbf{0}=0 \quad \text { and } \quad \mathbf{1}=1
$$

and

$$
x \wedge y=x+y, \quad \mathbf{0}=1 \quad \text { and } \quad \mathbf{1}=0
$$

respectively.

We have thus proven our main result. 
Theorem. There are only two interpretations from $\mathbf{D}_{\mathbf{0 1}}$ into $\mathbf{B}_{\omega}$.

\section{INTERPRETING $\mathbf{B}_{\omega}$ IN $\mathbf{B}_{\omega}$}

All we have to do is to define a new pseudocomplement

$$
x^{\prime}:=\tau(x) \in \mathbf{F}_{\mathbf{B}_{\omega}}(\mathbf{x}),
$$

in such a way that for any $p$-algebra $\left\langle A ;+, \cdot,{ }^{*}, 0,1\right\rangle$, defining $\vee, \wedge, 0$ and $\mathbf{1}$ as in the previous section, $\left\langle A ; \vee, \wedge,{ }^{\prime}, \mathbf{0}, \mathbf{1}\right\rangle$ is also a $p$-algebra.

It is clear that $x^{\prime}$ is not constant, so recalling that

$$
x \wedge x^{\prime}=\mathbf{0}
$$

a quick check at the following table

\begin{tabular}{ccc}
$x^{\prime}$ & $x+x^{\prime}$ & $x \cdot x^{\prime}$ \\
\hline$x$ & $x$ & $x$ \\
$x^{*}$ & $x+x^{*}$ & 0 \\
$x^{* *}$ & $x^{* *}$ & $x$ \\
$x+x^{*}$ & $x+x^{*}$ & $x$ \\
$x^{*}+x^{* *}$ & $x^{*}+x^{* *}$ & $x$
\end{tabular}

shows the following:

Theorem. The only interpretation of $\mathbf{B}_{\omega}$ into itself is the trivial interpretation.

\section{REFERENCES}

1. R. Balbes and Ph. Dwinger, Distributive lattices, Univ. Missouri Press, Columbia, 1974.

2. J. Berman and $\mathrm{Ph}$. Dwinger, Finitely generated pseudocomplemented distributive lattices, J. Austral. Math. Soc. Ser. A 19 (1975), 239-246.

3. O. C. Garcia and W. F. Taylor, The lattice of interpretability types of varieties, Mem. Amer. Math. Soc., no. 305, Amer. Math. Soc., Providence, RI, 1984.

4. W. D. Neumann, On Malt'sev conditions, J. Austral. Math. Soc. Ser. A 17 (1974), 376-384. CHILE

Pontificia Universidad Católica de Chile, facultad de Matemáticas, Sede, Temuco,

Tupungato 482, Depto. C, Temuco, Chile

Pontificia Universidad Católica de Chile, Facultad de Matemáticas, Casilla 6177, Santiago, Chile

Llanquihue 01785, Temuco, Chile 